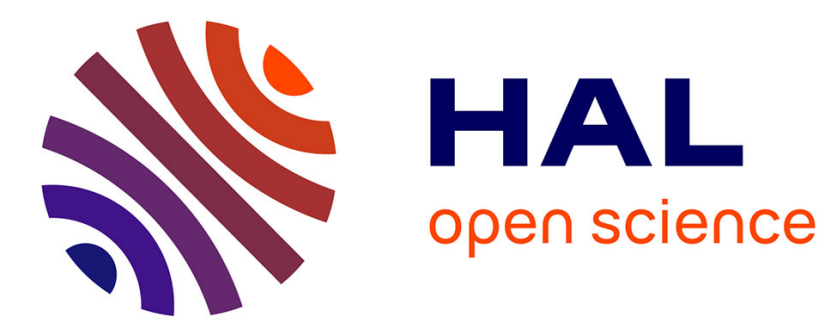

\title{
Unsteady heated vertical channel flow in a cavity
}

Yiqin Li, Hervé Pabiou, Christophe Ménézo

\section{To cite this version:}

Yiqin Li, Hervé Pabiou, Christophe Ménézo. Unsteady heated vertical channel flow in a cavity. International Journal of Thermal Sciences, 2018, 125, pp.293-304. 10.1016/j.ijthermalsci.2017.11.023 . hal-01708465

\section{HAL Id: hal-01708465 \\ https://hal.science/hal-01708465}

Submitted on 13 Feb 2018

HAL is a multi-disciplinary open access archive for the deposit and dissemination of scientific research documents, whether they are published or not. The documents may come from teaching and research institutions in France or abroad, or from public or private research centers.
L'archive ouverte pluridisciplinaire HAL, est destinée au dépôt et à la diffusion de documents scientifiques de niveau recherche, publiés ou non, émanant des établissements d'enseignement et de recherche français ou étrangers, des laboratoires publics ou privés. 


\title{
Unsteady heated vertical channel flow in a cavity
}

\author{
Yiqin Li ${ }^{\mathrm{a}, \mathrm{b}}$, Hervé Pabiou ${ }^{\mathrm{a}, *}$, Christophe Ménézo ${ }^{\mathrm{b}}$ \\ ${ }^{a}$ Univ Lyon, CNRS, INSA-Lyon, Université Claude Bernard Lyon 1, CETHIL UMR5008, F-69621, Villeurbanne, France \\ ${ }^{b}$ Université Savoie Mont-Blanc, LOCIE UMR CNRS 5271, Campus Scientifique Savoie Technolac - Bâtiment Helios, \\ Avenue du Lac Leman, F-73376, Le Bourget-du-Lac, France
}

\begin{abstract}
This experimental work investigates buoyant flow in a differentially heated vertical channel located inside a water cavity. The flow is found to be highly unsteady, and the key aspect of this study is to consider this unsteady behavior as a succession of states that turn out to be driven by the flow outside the channel. A conditional mean operator with respect to the average wall temperature is used to disentangle the different states through which the flow passes. Most of these states are characterized by a transition from laminar heat transfer in the bottom part of the channel to turbulent heat transfer, with a transition point that moves toward the exit as the average wall temperature increases. For the highest values of the average wall temperature, no transition is observed, and the heat exchange is found to be similar to that along a single vertical plate. For an intermediate range of wall temperature, a transition zone with turbulent heat transfer is observed in the upper part of the channel, and the heat transfer is found to follow the same laws as found for a symmetrically heated channel. For the lowest values of the wall temperature, the beginning of the turbulent zone is observed near the entry. The analysis is extended to several channel widths. The origin of the unsteady behavior is attributed to the flow in the whole cavity, and the conditional mean operator allows characterization of the flow inside the channel independently from the flow in the surroundings.
\end{abstract}

Keywords: Natural convection, Turbulent heat transfer, Unsteady flow, Vertical open channel, Conditional mean operator

PACS: 44.25.+f, 44.15.+a, 47.27.te

\section{Introduction}

Natural convection has been widely studied for passive cooling of electronics components and optimization of cooling fins, and its properties in these situations are now fairly well understood. However, natural convection in larger structures such as double-skin façades on buildings poses a much trickier problem, since the flow exhibits transitional or turbulent regimes that are less well understood. A typical configuration for convection around vertical geometries is that of natural convection in an open vertical channel with wall heating in infinite surroundings. In that case, one may define a modified Rayleigh number to characterize the flow: $R a_{b}^{*}=g \beta q_{\mathrm{w}} b^{5} / \lambda \kappa_{\kappa} H$,

\footnotetext{
*Corresponding author

Email address: herve.pabiou@insa-lyon.fr (Hervé Pabiou) 
where $g$ is the gravitational acceleration, $q_{\mathrm{w}}$ the wall heat flux, $b$ and $H$ the width and height of the channel, and $\beta, v, \lambda$, and $\kappa$ the volumetric thermal expansion coefficient, kinematic viscosity, thermal conductivity, and thermal diffusivity of the fluid, respectively. The Rayleigh number compares the buoyancy term that tends to lift the fluid with the viscous and thermal diffusive terms that tend to slow it down. Natural convection flow in vertical channels has been intensively investigated for many years for applications at low Rayleigh number $\left(R a_{b}^{*}<10^{5}\right)$ [1]. However, there has been little investigation of high-Rayleigh-number cases, which are appropriate for applications such as photovoltaic double-skin façades $\left(R a_{b}^{*} \sim 10^{10}\right)$.

For high-Rayleigh-number natural convection flow, transitions from laminar to turbulent flow have been reported. Miyamoto et al. [2] studied a $5 \mathrm{~m}$-high air channel heated on one wall with an isoflux condition, the other wall being adiabatic. The wall temperature and velocity were measured for several aspect ratios $(H / b=100,50$, and 25). The maximum wall temperature was located between 1 and $2 \mathrm{~m}$ above the channel inlet for modified Rayleigh numbers between $2 \times 10^{4}$ and $2 \times 10^{7}$. The authors attributed this maximum to a transition from laminar to turbulent flow. A few years later, Webb and Hill [3] studied natural convection flow in a vertical air channel under an isoflux heating condition on one wall with adiabatic extensions and an adiabatic condition on the other walls. Modified Rayleigh numbers ranging from 503 to $1.75 \times 10^{7}$ were reached. The authors found no indication of transition to turbulent heat transfer in any of their experiments. A correlation between the local Nusselt number and the local Grashof number for modified Rayleigh numbers below $10^{7}$ was found. More recently, a transition has been observed by Daverat et al. [4] on the same experimental bench as used in the present study but with a symmetrical heating configuration. A $611 \mathrm{~mm}$-high and $45 \mathrm{~mm}$-wide water channel was heated with a uniform heat flux on both walls to reach $R a_{b}^{*}=1.7 \times 10^{6}-4.3 \times 10^{7}$. A change in flow behavior was observed at $R a_{b}^{*}=10^{7}$. Detailed observations of the behavior on the same experimental bench have been reported in a recent work by the same team [5]. The channel was heated on its two walls with a uniform heat flux $q_{w}=1150 \mathrm{~W} \mathrm{~m}^{-2}$; its width was $b=59 \mathrm{~mm}$, giving a modified Rayleigh number $R a_{b}^{*}=6.7 \times 10^{7}$. Temperature and velocity measurements in several sections of the channel showed that this change in behavior corresponded to a transition from laminar heat transfer in the bottom part of the channel to turbulent heat transfer in the upper part. More precisely, below $x / H=0.71$, most of the heat was transported in the conductive sublayer, whereas above $x / H=0.71$, a significant part of the heat was transferred by fluctuations to the center of the channel, leading to a sudden increase in the temperature of the bulk flow. This transition is analyzed in detail by Li et al. [6] through a scaling analysis. Assuming a two-dimensional mean flow, the half-channel is split into seven zones where the momentum and energy equations are reduced to their leading terms. The heat transfer in the bottom part is shown to be similar to that along a single heated vertical plate with a wall temperature that obeys the following power law [7]:

$$
\left\langle\Delta T_{w}\right\rangle_{t}(x)=\left\langle\Delta T_{w, T}\right\rangle_{t}\left(\frac{x}{\left\langle x_{T}\right\rangle_{t}}\right)^{1 / 5} \quad\left(x \leq\left\langle x_{T}\right\rangle_{t}\right)
$$

where $x$ is the distance to the entry, $x_{T}(t)$ is the height of the transition, $\Delta T_{w}(x, t)=T_{w}(x, t)-$ $T_{\text {entry }}(t), T_{w}(x, t)$ is the local wall temperature, $T_{\text {entry }}(t)$ is the temperature at the entry and \langle\rangle$_{t}$ is the time-average operator, $\Delta T_{w, T}=\Delta T_{w}\left(x_{T}, t\right)$. In the upper part, $\alpha q_{w}$ denotes the horizontal heat flux through the edge of the conductive sublayer toward the center of the channel. The decrease in wall temperature above the transition is found to be well approximated by the following equation: 


$$
\left\langle\Delta T_{w}\right\rangle_{t}(x)=\left\langle\Delta T_{w, T}\right\rangle_{t}\left(\alpha+(1-\alpha) \frac{x}{\left\langle x_{T}\right\rangle_{t}}\right)^{1 / 5} \quad\left(x \geq\left\langle x_{T}\right\rangle_{t}\right)
$$

with $\alpha=2.6$. This decrease in wall temperature is a consequence of a transition from laminar to turbulent heat transfer. More precisely, Eq. (2) models a transition zone, and the fully turbulent regime with an increasing wall temperature is not observed in this study. These works show that, for a symmetrically heated channel, the transition is triggered by the meeting of the two shear layers that develop in the left and right half-channels between the velocity peaks close to the walls and the center of the channel. By comparison, Liu and Vliet [8] observed the beginning of the transition zone in a vertical flat-plate configuration in a water flow at $R a_{x}=10^{13}$, where $R a_{x}$ is defined by Eq. (8a). In the study by Daverat et al. [5], the Rayleigh number at the exit of the channel, $R a_{H}$, is slightly less than $10^{13}$, indicating that in a symmetrically heated vertical channel, the transition point is moved down with respect to the single-plate configuration.

Furthermore, flow reversals re-entering the channel from the outlet have been observed in several studies with asymmetrical wall heating. Sparrow et al. [9] performed an experimental study of flow reversals in a $14.5 \mathrm{~cm}$-high water channel with an isothermal condition on one wall. They observed a V-shaped flow reversal close to the unheated wall near the outlet of the channel. For three different channel widths, a $N u-R a$ correlation was established. It is interesting to note that this correlation is similar to that found for a single vertical heated plate at uniform temperature by Ostrach [10]. Sanvicente et al. [11] observed unsteady flow reversals in a $1.5 \mathrm{~m}-$ high air channel with isoflux heating on one wall, the other wall being adiabatic. They suggested that these structures originated from fluctuations in the surroundings. Flow reversals have also been studied by Dupont et al. [12], who used a $600 \mathrm{~mm}$-high air channel with asymmetrical heating condition. They classified the flow into three states: no reversal, intermittent reversals, and permanent reversal. Their results also showed that the width of the recirculation zone was proportional to the width of the channel and independent of the Rayleigh number. Recently, Fu et al. $[13,14]$ performed a numerical study of flow reversal in an asymmetrically heated channel with constant temperature and adiabatic boundary conditions. They found that flow reversals occur more frequently for large channel widths and that they are strongly linked with the pressure distribution at the outlet. At present, it is not known whether or not these observations also hold for isoflux conditions.

In addition to studies of flow reversal over long durations, transient development of reversal at the beginning of heating has also been investigated. Polidori et al. [15] investigated transient flow in a vertical water channel with the same configuration as in Webb and Hill's study. They observed flow structures during the first few minutes after the onset of heating. Flow visualization showed that reversed flow was established after 5 minutes of heating, and velocity measurements revealed its unsteady nature. This study highlighted the fact that the recirculation zone may reach the entry of the heated zone. The same team [16] also visualized flow after 50 minutes of heating and measured the length of the recirculation zone as a function of the Rayleigh number and the aspect ratio $H / b$. A figure-of-eight shaped structure was used to describe the reversed flow. These authors observed that the overall length of the structure decreased with increasing Rayleigh number or aspect ratio.

In a numerical study by Garnier et al. [17], natural convection flow was shown to develop in an open vertical channel with asymmetrical isoflux heating, within enclosures of different sizes. At the onset of heating, the fluid flowed out of the channel like a plume that evolved in time and space. These numerical results indicated that the direction of the plume jet and the enclosure 
size both had a strong influence on the global recirculation in the channel. Indeed, the hot fluid that flows out of a heated channel can be viewed as a thermal plume. Atmane et al. [19] studied the phenomenon of plume oscillation in a natural convection flow around a vertically confined and horizontal heated cylinder. They conducted temperature and velocity measurements on an experimental bench with water as the fluid. The plume was observed to move from one side of the vertical axis of the cylinder to another. The authors suggested that the oscillations of the plume could result from interactions between the two secondary vortices above the cylinder and the free surface, since horizontal displacement of one of the vortices will induce movement of the vertical plume to one side or another.

It is clear from this literature review that the transition to turbulence and of flow reversal in natural convection in vertical channels are still not fully understood.

The objective of the present work is to study heat transfer in an asymmetrically heated channel with isoflux boundary conditions inside a finite cavity. As the flow turns out to be unsteady, the aim is to provide a heat transfer model in an unsteady flow, to highlight the effect of the finite size of the cavity and to understand the influence of the aspect ratio of the channel. The paper is organized as follows. After a description of the experimental apparatus, the buoyant flow is characterized and a global picture of the flow is given. The unsteady heat transfer is analyzed using a conditional mean operator, and a heat transfer model is presented. The influence of the flow that develops outside of the channel is then highlighted. Finally, different aspect ratios are studied. The final part of the paper is devoted to a discussion of the origin of flow unsteadiness.

\section{Nomenclature}

$b \quad$ Channel width (m)

$B(g) \quad$ Fixed error of a quantity $g$ (units are those of $g$ )

$g \quad$ Acceleration due to gravity $\left(\mathrm{m} \mathrm{s}^{-2}\right)$

$H \quad$ Channel height (m)

$l \quad$ Channel depth (m)

$N u_{x} \quad$ Local Nusselt number, Eq. (8b)

$P_{e} \quad$ Mean pressure outside the channel (Pa)

$\hat{P}_{e} \quad$ Mean driving pressure outside the channel (see Appendix B) (Pa)

$q_{w} \quad$ Mean wall heat flux $\left(\mathrm{W} \mathrm{m}^{-2}\right)$

$q_{c, \text { sym }}$ Turbulent vertical heat flux outside the conductive thermal boundary layer in the top part of the channel in a symmetrically heated channel (see Eq. [42] in [6]) $\left(\mathrm{W} \mathrm{m}^{-2}\right)$

$R a_{b}^{*} \quad$ Modified Rayleigh number, $R a_{b}^{*}=g \beta q_{\mathrm{w}} b^{5} / \lambda v \kappa H$

$R a_{x} \quad$ Local Rayleigh number, Eq. (8a)

$S \quad$ External stratification parameter, Eq. (11)

$t \quad$ Time (s)

$T \quad$ Temperature (K)

$U, V \quad$ Mean velocity component in the $x, y$ directions, respectively $\left(\mathrm{m} \mathrm{s}^{-1}\right)$

$\overrightarrow{U_{e}} \quad$ Mean velocity vector outside the channel $\left(\mathrm{m} \mathrm{s}^{-1}\right)$

$\overrightarrow{u_{e}^{\prime}} \quad$ Velocity fluctuation vector outside the channel $\left(\mathrm{m} \mathrm{s}^{-1}\right)$

$U_{I} \quad$ Typical velocity of laminar flow along a vertical flat plate $\left(\mathrm{m} \mathrm{s}^{-1}\right)$

$u^{\prime}, v^{\prime} \quad$ Instantaneous velocity fluctuations in the $x, y$ directions, respectively 


\begin{tabular}{|c|c|}
\hline$x$ & Distance from inlet in the ascending direction $(\mathrm{m})$ \\
\hline$x_{T}(t)$ & Instantaneous height of the transition \\
\hline$y$ & Distance from the left wall $(\mathrm{m})$ \\
\hline$Y_{U=0}$ & $\begin{array}{l}\text { Distance from the heated wall of the position of zero vertical velocity } \\
\text { at } x / H=0.75(\mathrm{~m})\end{array}$ \\
\hline$Y_{\text {cell }}$ & $\begin{array}{l}y \text { position of the plane separating contrarotating cells located imme- } \\
\text { diately above the channel }(\mathrm{m})\end{array}$ \\
\hline$z$ & Distance from the front lateral wall $(\mathrm{m})$ \\
\hline$\alpha q_{w}$ & $\begin{array}{l}\text { Horizontal heat flux through the conductive sublayer in the top part } \\
\text { of the channel }\left(\mathrm{W} \mathrm{m}^{-2}\right)\end{array}$ \\
\hline$\beta$ & Isobaric thermal expansion coefficient of water $\left(\mathrm{K}^{-1}\right)$ \\
\hline$\delta_{V}$ & conductive thermal boundary-layer thickness in the bottom part $(\mathrm{m})$ \\
\hline$\theta_{w}(x)$ & Reduced wall temperature, Eq. (4) (K) \\
\hline$\Gamma=H / b$ & Aspect ratio \\
\hline$\Delta T_{w}(x)$ & Mean wall temperature difference at the entry of the channel $(\mathrm{K})$ \\
\hline$\Delta T_{I}$ & $\begin{array}{l}\text { Typical temperature difference with respect to the inlet temperature } \\
\text { of laminar flow along a vertical flat plate }(\mathrm{K})\end{array}$ \\
\hline $\mathcal{E}(g)$ & Uncertainty of a quantity $g$ (units are those of $g$ ) \\
\hline$\kappa$ & Thermal diffusivity of water $\left(\mathrm{m}^{2} \mathrm{~s}^{-1}\right)$ \\
\hline$\lambda$ & Thermal conductivity of water $\left(\mathrm{W} \mathrm{m}^{-1} \mathrm{~K}^{-1}\right)$ \\
\hline$v$ & Kinematic viscosity of water $\left(\mathrm{m}^{2} \mathrm{~s}^{-1}\right)$ \\
\hline$\rho$ & Density of water at the reference temperature $\left(\mathrm{kg} \mathrm{m}^{-3}\right)$ \\
\hline \multicolumn{2}{|l|}{ Operators } \\
\hline$\sigma_{g}$ & Root mean square of a quantity $g$ (units are those of $g$ ) \\
\hline$\langle\cdot\rangle_{\xi}$ & Averaging operator over a variable $\xi$ \\
\hline$\left\langle\cdot \mid \theta_{w}^{s}\right\rangle$ & Conditional mean operator, Eq. (5) \\
\hline$\nabla$ & Partial derivative operator \\
\hline 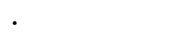 & Dot product of two vectors \\
\hline$\otimes$ & Tensor product of two vectors \\
\hline \multicolumn{2}{|l|}{ Subscripts } \\
\hline inlet & Referring to channel inlet \\
\hline$e$ & Referring to the surroundings of the channel \\
\hline $\max$ & Referring to the maximum \\
\hline$H$ & Referring to the channel outlet \\
\hline$T$ & Referring to the height of the transition \\
\hline$w$ & Referring to the heated wall \\
\hline$x<x_{T}$ & Referring to the bottom part of the channel \\
\hline$x>x_{T}$ & Referring to the top part of the channel \\
\hline
\end{tabular}

\section{Experimental apparatus}

A natural convection flow is achieved in a vertical water channel heated on one wall, the other wall being adiabatic. The experimental set-up is the same as the one used in Daverat et al. [4], except that, in this study, the vertical heated plate is made of stainless steel and the adiabatic one 


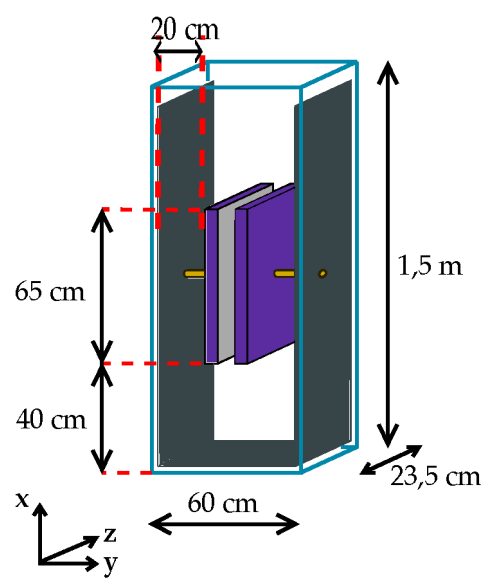

(a)



(b)

Figure 1: (a) Schematic illustration of the experimental apparatus. (b) Detailed view of the apparatus. The positions of the thermocouples are indicated by the markers $\bullet$. The insulation of the glass tank and the cooling system are not shown.

of polycarbonate. Another difference is that the flow velocity is measured using particle imaging velocimetry (PIV) rather than laser Doppler velocimetry.

The apparatus is made of two waterproof boxes immersed in a water tank as shown in Fig. 1. One side of each box, which is the heated or adiabatic wall of the channel, is stuck on a polycarbonate frame and the other side, made of thick polycarbonate, is screwed onto the frame. Twelve electrical heaters are stuck onto the back of each channel wall (the heaters stuck onto the polycarbonate plate are disconnected in this study) and insulating material is added to the back of the heaters to reduce heat loss (each heater is equipped with a heat flux meter to measure losses from its back). The depth ( $z$ direction; see Fig. 1a) of each waterproof box $(l=230 \mathrm{~mm})$ is equal to the internal width of the tank, so the lateral walls of the channel are the glass walls of the tank. The distance between the two waterproof boxes, which is the channel width $b$, is adjustable between 0 and $14 \mathrm{~cm}$. The lateral walls of the tank are covered by a $5 \mathrm{~cm}$-thick layer of insulating material with a thermal conductivity of $0.033 \mathrm{~W} \mathrm{~m}^{-1} \mathrm{~K}^{-1}$, except for one lateral wall of the channel, which is left uncovered to allow velocity measurements. The water outside the channel is cooled with an external thermal regulation system that pumps hot water far from the channel and discharges cold water near the exit of the channel (see "Water suction" and "Water discharge" in Fig. 1b). The cooling loop consists of a bath and a pump with variable flow rate. The temperature of the room in which the experimental set-up is located is controlled within $\pm 2 \mathrm{~K}$.

$x$ is the ascending vertical, $y$ is the distance to the heated plate, and $z$ is the horizontal axis parallel to the heated plate. The origin of the vertical axis $x=0$ is defined as the beginning of the heated zone. Heat losses through the lateral walls (the $x O y$ planes) are estimated through a specific experiment. The exit of the channel is closed by a foam block so that a significant part of the injected power is dissipated through the lateral walls. A basic thermal model is built and thermal resistance are identified by using measurements of several experiments. This method 
leads to an uncertainty around $\pm 2 \%$ of the injected heat flux. Thus, an adiabatic boundary condition is assumed for the lateral walls of the channel. Thermocouples have been added to the bench described in Daverat et al. [4]. Wall temperatures are measured at the center of each heater and between every two heaters. More precisely, 25 thermocouples are embedded in each wall along the central axis $(z / l=0.5)$ as shown in Fig. 2. Temperatures are also measured outside the channel by thermocouples located along the two stems behind each wall (Fig. 2). All thermocouples are calibrated by using an in-house system that leads to an uncertainty of $\pm 0.1^{\circ} \mathrm{C}$ in the temperature measurement. Velocity fields are obtained using a PIV system consisting of an Nd:YAG

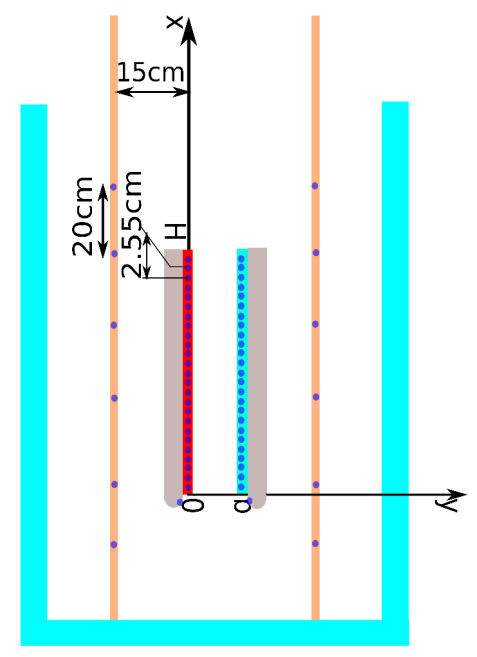

Figure 2: Positions of the thermocouples. The left stainless-steel wall is heated with a constant heat flux. The right polycarbonate wall is adiabatic.

double-cavity laser and a CCD camera. The seeding tracers are $20 \mu \mathrm{m}$ polyamide particles with a mean density of $1.4 \mathrm{~kg} \mathrm{~m}^{-3}$. A laser sheet illuminated from the top of the water tank is obtained in the central vertical plane of the channel $(z / l=0.5)$. The camera in front of the water tank records images at the outlet level of the channel with an observation window of $281 \times 281 \mathrm{~mm}^{2}$ (including the last $180 \mathrm{~mm}$ of the channel height and $100 \mathrm{~mm}$ above). A double-frame recording with a time interval of $65000 \mu \mathrm{s}$ is taken for a duration of $10 \mathrm{~h}$ at a frequency of $0.2 \mathrm{~Hz}$. The recorded film is post-processed and velocity fields are obtained using DaVis LaVision software. The uncertainty on velocity comes from three sources: image calibration, sedimentation of tracing particles, and uncertainties in particle positions due to lack of image resolution. Concerning the calibration process, the identification of a known distance on the image of a test pattern leads to an uncertainty of $\pm 0.1 \mathrm{~mm} . \mathrm{s}^{-1}$. The effect of sedimentation is estimated through the Stoke velocity of a particle which induces an uncertainty of $\pm 0.1 \mathrm{~mm} . \mathrm{s}^{-1}$. The largest uncertainty comes from the identification of the position of the particle in the $32 \times 32$ pixels $^{2}$-correlation-windows which leads to uncertainty of $\pm 0.5 \mathrm{~mm} . \mathrm{s}^{-1}$. Following Ref. [18], these independent uncertainties are combined through the root-sum-square method and the overall uncertainty in the velocity is estimated as $\pm 0.5 \mathrm{~mm} \mathrm{~s}^{-1}$. All temperature and velocity measurements are made in the central 
plane $x O y$ of the channel at $z / l=0.5$. The acquisition rate for temperature and heat-flux measurements is $0.17 \mathrm{~Hz}$. The main uncertainties are $\pm 2 \%$ in the injected heat flux, $\pm 0.1{ }^{\circ} \mathrm{C}$ in the temperature, and $\pm 0.5 \mathrm{~mm}$ in the location of the thermocouples embedded in the heated walls.

The experiment presented in this study lasts more than $300 \mathrm{~h}$, but we only consider the range $t=190-240 \mathrm{~h}$ during which the flow rate of the cooling loop is fixed at $1.8 \mathrm{~L} \mathrm{~min}^{-1}$. As already mentioned, the average temperature of the cavity is controlled by a cooling system that injects temperature-regulated water through diffusers near the exit of the channel (Fig. 1b). Several flow rates of the cooling system have been tested over more than $300 \mathrm{~h}$ to check whether it modifies the long-term behavior. As explained below, the wall temperature is always unsteady over long times, and, as far as it is possible to compare such flows, no change in heat transfer could be attributed to the cooling system. With regard to local disturbances created by the injection of cold water, it is important to note that we use a geared pump that is known to produce weak disturbances. Incidentally, the typical timescale of the fluctuations of the cooling system is much smaller than the typical timescale for measurement of the wall temperature and cannot be responsible for the unsteady flow described below.

The results given in Sections 3.1-3.4 correspond to a configuration characterized by a channel width $b=40.6 \mathrm{~mm}$ and a heat flux $q_{w}=3677 \mathrm{~W} \mathrm{~m}^{-1}$ to reach a modified Rayleigh number $R a_{b}^{*}=3.2 \times 10^{7}$, the thermophysical properties being taken at $T_{\text {ref }}=34^{\circ} \mathrm{C}$. This reference temperature corresponds to $T_{\text {ref }}=\left(\left\langle T_{\text {entry }}\right\rangle_{t}+\left\langle T_{w}\right\rangle_{t, \max }\right) / 2$, where $\left\langle T_{w}\right\rangle_{t \text {, max }}$ is the maximum of the time-averaged temperature of the heated wall. The measurements analyzed in this study correspond to a range where the temperature at the entry reaches a quasi-steady state. The $10 \mathrm{~h}$ long velocity measurement by PIV corresponds to the period $t=220.1-230.1 \mathrm{~h}$. A comparison with other experiments at different widths is presented in Section 3.5.

\section{Results}

\subsection{Phenomenological description of the flow behavior}

Fig. 3 shows the time evolution of $\left\langle\Delta T_{w}\right\rangle_{x}(t)$, where \langle\rangle$_{x}$ is the $x$-average operator, $\Delta T_{w}(x, t)$ being measured in the symmetry plane $z / l=0.5$ (see Fig. 2 for the distribution of the thermocouples). At first glance, a particular behavior with small fluctuations around a high value can be seen between $t=222.7 \mathrm{~h}$ and $t=223.4 \mathrm{~h}$. For convenience, this particular behavior is hereinafter referred to as the single-plate mode, the name being justified in the following sections. To get an approximate quantification, a rough criterion, which is used only in this section, is given by

$$
\left\langle\Delta T_{w}\right\rangle_{x}(t) \geq\left\langle\Delta T_{w}\right\rangle_{x, t}+\sigma_{\left\langle\Delta T_{w}\right\rangle_{x}}
$$

where $\sigma_{\left\langle\Delta T_{w}\right\rangle_{x}}$ is the root mean square (RMs) value of $\left\langle\Delta T_{w}\right\rangle_{x}$. This criterion defines a threshold for the average wall temperature above which the flow is considered to be in this particular state. Using this criterion, the single-plate mode appears several times, with durations ranging from a few minutes to almost one hour.

In order to link this thermal behavior with the velocity fields, the evolution of the vertical velocity measured by PIV near the outlet of the channel is compared with the evolution of the wall temperature at the same time $(t=220.1-230.1 \mathrm{~h})$. Fig. 4 shows the evolution of the wall temperature and of the instantaneous velocity profile at $x / H=0.75$. The most obvious appearance of the single-plate mode observed on the temperature (see Fig. 4a between $t=222.7 \mathrm{~h}$ and $t=223.4 \mathrm{~h}$ ) seems to be associated with negative velocities reaching $-5 \mathrm{~mm} \mathrm{~s}^{-1}$ in a zone that occupies more than half of the channel width. In terms of the penetration depth of this reversed 


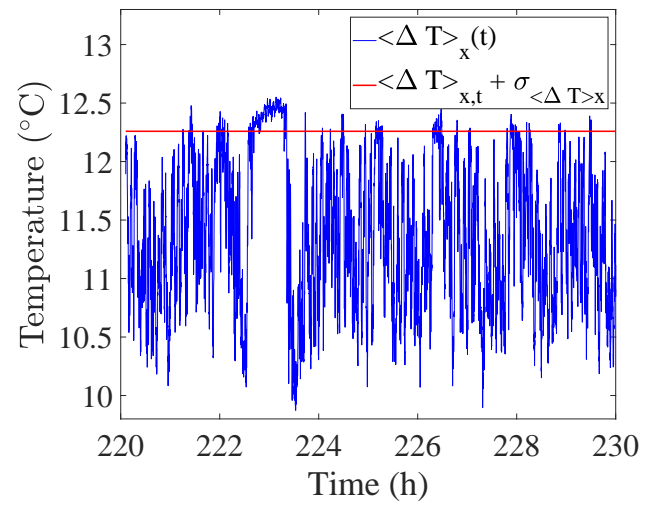

Figure 3: Time evolution of the average wall temperature over a limited timespan corresponding to the velocity measurement time. The horizontal line represents $\left\langle\Delta T_{w}\right\rangle_{x, t}+\sigma_{\left\langle\Delta T_{w}\right\rangle_{x}}$.

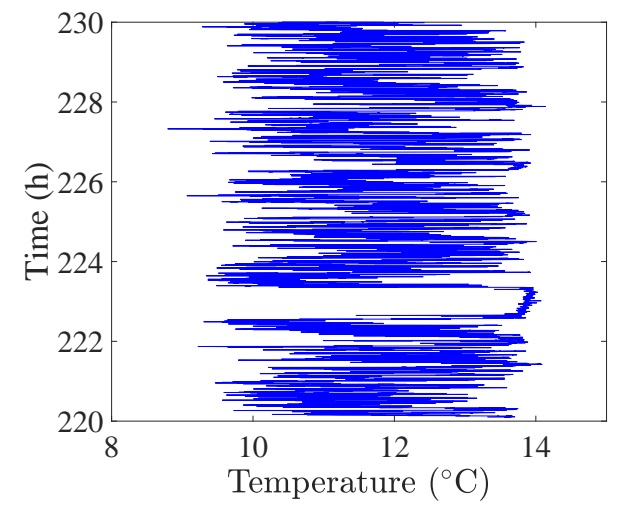

(a)

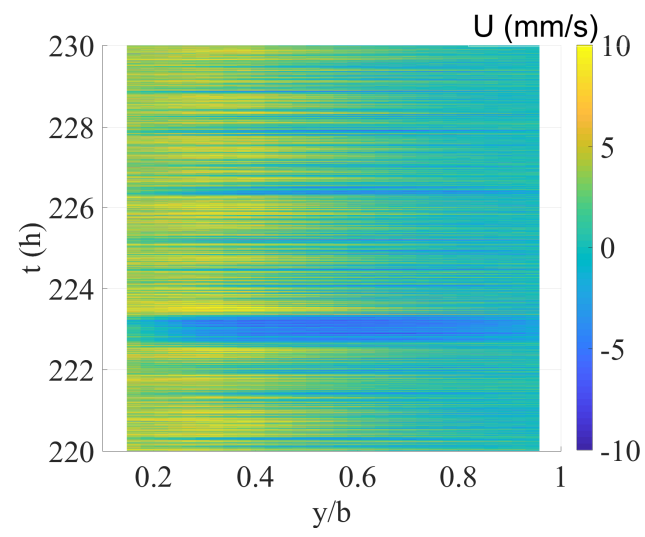

(b)

Figure 4: (a) Evolution of the wall temperature at $x / H=0.75$ for the time period $t=220.1-230.1 \mathrm{~h}$ during which velocities are measured. (b) Temporal evolution of the instantaneous vertical velocity profile averaged over $0.73 \leq$ $x / H \leq 0.77$ for $t=220.1-230.1 \mathrm{~h}$. 


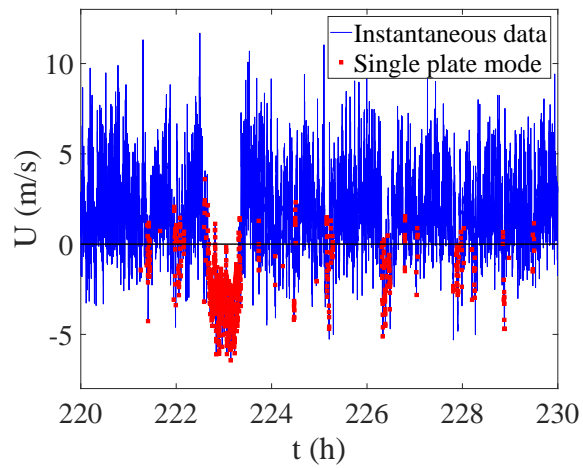

Figure 5: Vertical component of the bulk velocity averaged over $0.73 \leq x / H \leq 0.77$ and $0 \leq y / b \leq 1$ as a function of time. Red squares correspond to the single-plate mode according to Eq. (3).

flow, PIV velocity images provide a visualization only of the upper part of the channel above $x / H=0.7$. However, the velocity fields show that the large-scale reversed flow that occurs during the single-plate mode reaches at least to $x / H=0.7$.

The longest appearance of the single-plate mode around $t=222.7-223.4 \mathrm{~h}$ is accompanied by a flow reversal persisting for this entire duration. For other shorter appearances, Fig. 5 shows the vertical velocity averaged over $0.73 \leq x / H \leq 0.77$ and over the section $0 \leq y / b \leq 1$ as a function of time as well as the moments corresponding to the appearance of the single-plate mode according to Eq. (3). The appearance of the single-plate mode corresponds to the lowest values of the bulk velocity. However, it is worth noting that there are several negative velocities without the presence of the single-plate mode (e.g., $t \approx 220.3 \mathrm{~h}$ ). More generally, negative instantaneous bulk velocities are, most of the time, observed during the single-plate mode in Fig. 5, and similar observations are made at other positions $x / H$. Indeed, the flow rate seems to vary in time, with observations of negative bulk velocity during very short times and of persistent negative velocity sequences that are identifiable as the single-plate mode. Therefore, one can see the flow behavior as continuously evolving but sometimes locked in the single-plate mode. This description is explored in detail in Section 3.2.

\subsection{Global picture of the flow}

To obtain a more detailed description of the unsteadiness, a conditional mean operator, based on the average wall temperature, is defined as the mean value of a quantity over a subset of times for which a given condition is fulfilled. Let $\theta_{w}$ denote the reduced wall temperature:

$$
\theta_{w}(t) \doteq \frac{\left\langle\Delta T_{w}\right\rangle_{x}-\left\langle\Delta T_{w}\right\rangle_{x, t}}{\sigma_{\left\langle\Delta T_{w}\right\rangle_{x}}}
$$

The conditional mean $\left\langle g \mid \theta_{w}\right\rangle$ of a given quantity $g$ with respect to $\theta_{w}$ is defined as follows (see Chapter 12 in [20] for a rigorous definition):

$$
\left\langle g \mid \theta_{w}=\theta\right\rangle \doteq\left\langle g(t) \text { such that }\left|\theta_{w}(t)-\theta\right|<\mathrm{d} \theta / 2\right\rangle
$$

where \langle\rangle is the arithmetic mean operator and $\mathrm{d} \theta$ is a small step chosen to be consistent with the resolution of the temperature measurement, which is around $0.2^{\circ} \mathrm{C}$ in this study. In the same 
(a)

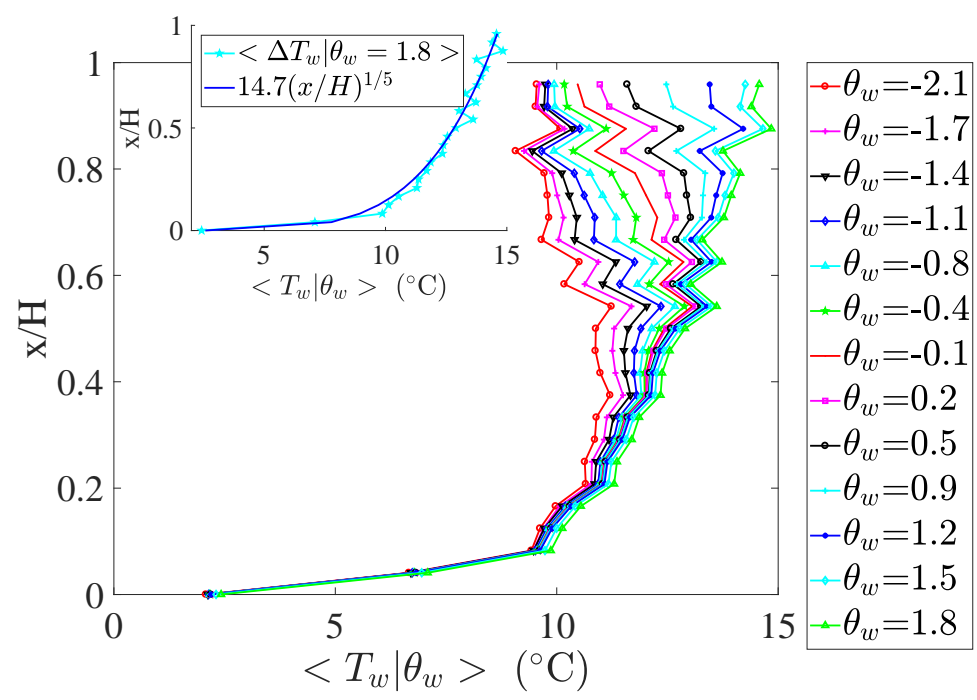

(b)

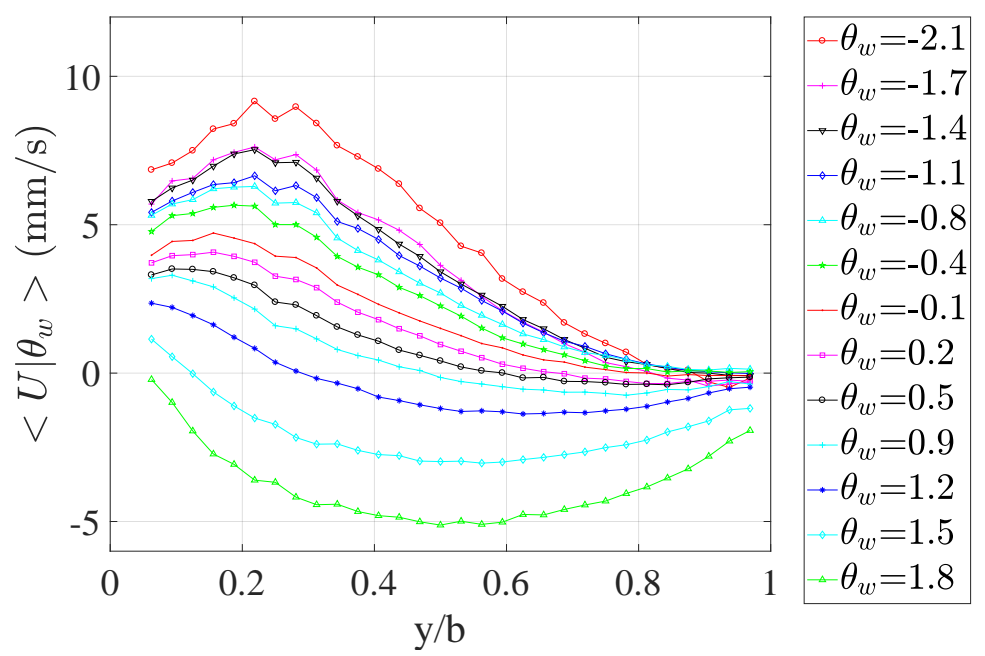

Figure 6: Conditional mean: (a) wall temperature and (b) vertical velocity component averaged over $0.73 \leq x / H \leq 0.77$.

way, one can define the conditional RMs value of a quantity by replacing the arithmetic mean operator in Eq. (5) by the standard deviation operator. To retrieve the time-average value from the conditional mean, one needs to know the probability density function (pdf) of $\theta_{w}$ :

$$
\langle g\rangle_{t}=\int_{\theta_{w}}\left\langle g \mid \theta_{w}\right\rangle \operatorname{pdf}\left(\theta_{w}\right) \mathrm{d} \theta_{w}
$$

This conditional mean allows one to split the unsteady signal with respect to the average wall temperature, and therefore one expects to find the single-plate mode identified in the previous section for $\theta_{w} \geq 1$ (see Eq. (3)). The relevancy of this parameter will be shown in the following sections. Fig. 6 shows the conditional mean profiles of wall temperature and of vertical velocity 




(a)

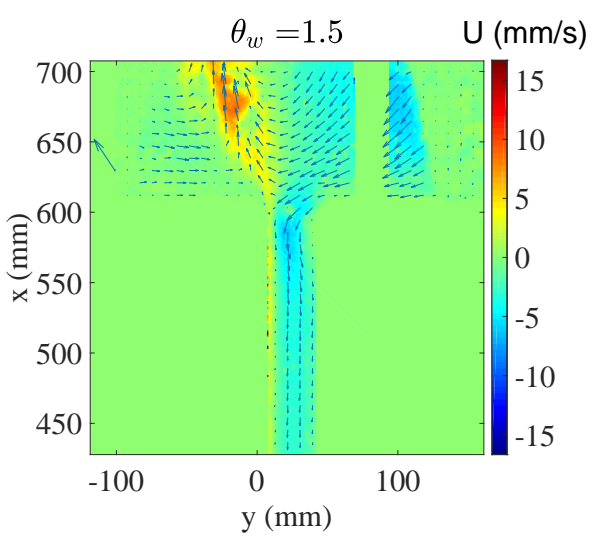

(b)

Figure 7: Conditional mean of the velocity field for (a) $\theta_{w}=-0.8$ and (b) $\theta_{w}=1.5$. The background color represents the vertical component $U$. The heated plate is on the left.

across the section, the velocity being averaged within $0.73 \leq x / H \leq 0.77$. Fig. 6 a clearly shows that for the highest values of $\theta_{w}\left(\theta_{w} \gtrsim 1\right)$, the wall temperature increases monotonically, whereas it exhibits a maximum value below the outlet for $\theta_{w} \lesssim 1$. The inset shows the experimental wall temperature for $\theta_{w}=1.8$, which is well fitted by the following one-fifth power law:

$$
\left\langle\Delta T_{w} \mid \theta_{w}=1.8\right\rangle=14.7\left(\frac{x}{H}\right)^{1 / 5}, \quad \text { Temperature in } \mathrm{K}
$$

This power law is identical to that obtained in the case of natural convection along a single vertical plate with isoflux boundary conditions [7], justifying the name of single-plate mode. For lower values of $\theta_{w}$, the wall temperature profile exhibits a maximum that moves toward the entry as $\theta_{w}$ decreases. Similarly, the vertical bulk velocity is a decreasing function of $\theta_{w}$ (Fig. 6b), and one observes a large flow reversal for $\theta_{w} \gtrsim 1$.

In order to give a picture of the flow, the conditional means of the velocity fields for $\theta_{w}=-0.8$ and $\theta_{w}=1.5$ are plotted in Fig. 7, where the background color shows the vertical velocity component. For conditional means corresponding to $\theta_{w} \geq 1.2$, the velocity fields show significant flow reversal along the adiabatic plate, whereas for lower values of $\theta_{w}$, the velocity fields look like that shown in Fig. 7a. In both figures, a pair of counter-rotating cells is observed above the channel outlet. The main difference between the fields above the channel shown in Figs. 7a and $7 \mathrm{~b}$ is the location of the plane separating the two cells. For low values of $\theta_{w}$ (as in Fig. 7a), the ascending hot fluid that flows out of the channel near the heated wall rises vertically above the channel between the two cells. For high values of $\theta_{w}$ (as in Fig. 7b), flow reversal can clearly be seen, with the two cells being moved to the left, which interferes with the hot fluid that flows out of the channel. Therefore, the bulk velocity fluctuations observed in Fig. 5 could be related to the uncontrolled movement of the cells above the channel as their shifting modifies the outlet conditions. In this way, the large-scale flow reversal during long periods could be seen as a particular situation where the right cell obstructs the entire exit section of the channel. Indeed, as the right cell has clockwise circulation, a left shift tends to favor the appearance of downward flow in the channel. In the case of a heated channel with a large flow reversal that extends to the entry, the fluid flows downward along the adiabatic wall before flowing upward. Therefore, 
in a vertical plane far from the heated plate, the tangents to the streamlines (i.e., the velocity directions) are horizontal, which creates the conditions for the existence of the single plate-type solution. This solution seems to be stable enough to be observed for long times. This point is discussed in Section 3.4.

\subsection{Heat transfer modeling}

As recalled in Section 1, a vertical channel with symmetrical heating exhibits a single-platelike heat transfer to a transition point above which turbulent heat transfer occurs and the wall temperature decreases. Fig. 6a indicates that similar behavior occurs for an asymmetrical heating configuration, with a transition point that moves toward the exit as the average wall temperature increases. To test this hypothesis, Eqs. (1) and (2) are first converted into dimensionless form by defining local Nusselt and Rayleigh numbers as follows:

$$
\begin{aligned}
R a_{x} & =\frac{g \beta q_{w} x^{4}}{\lambda v \kappa} \\
N u_{x} & =\frac{q_{w} x}{\lambda\left\langle\Delta T_{w}\right\rangle_{t}}
\end{aligned}
$$

Let $R a_{T}$ and $N u_{T}$ denote the Rayleigh and Nusselt numbers at the transition, which are defined as the values of $R a_{x}$ and $N u_{x}$ at the transition point $x=\left\langle x_{T}\right\rangle_{t}$. Then, the wall temperature correlations given in Eqs. (1) and (2) become

$$
\begin{array}{ll}
N u_{x<x_{T}}=N u_{T}\left(\frac{R a_{x}}{R a_{T}}\right)^{1 / 5} & \left(x \leq x_{T}\right) \\
N u_{x>x_{T}}=N u_{T}\left(\frac{\left(\frac{R a_{x}}{R a_{T}}\right)^{5 / 4}}{\alpha+(1-\alpha)\left(\frac{R a_{x}}{R a_{T}}\right)^{1 / 4}}\right)^{1 / 5} & \left(x \geq x_{T}\right)
\end{array}
$$

In the symmetrical configuration studied in [6], heat transfer is modeled by Eqs. (9) and depends on three parameters: $\left(\left\langle x_{T}\right\rangle_{t},\left\langle\Delta T_{w, T}\right\rangle_{t}, \alpha\right)$ or alternatively $\left(R a_{T}, N u_{T}, \alpha\right) . x=\left\langle x_{T}\right\rangle_{t}$ denotes the mean position of the transition from laminar to turbulent heat transfer and it is defined as the location of the maximum wall temperature. $\left\langle\Delta T_{w, T}\right\rangle_{t}$ is the wall temperature at the transition, which is defined as the maximum wall temperature. $\alpha$ is the portion of the heat transported from the near-wall region to the center of the channel defined in [6] for the symmetrically heated channel. Indeed, below the transition, all of the heat is transported inside the thermal boundary sublayer (TBsL), the thickness of which is defined by $\delta_{V}=\lambda\left\langle\Delta T_{w}\right\rangle_{t} / q_{w}$. Above the transition, $\alpha q_{w}$ is the rate of heat transfer from the TBsL to the center of the channel by fluctuations, which defines $\alpha$. Let us suppose that the same scenario applies in the case of an asymmetrically heated channel. More precisely, for a given average wall temperature, the flow exhibits a transition from a laminar heat flux in the bottom part to a turbulent one in the top part at a certain location $x_{T}$. In the bottom part, heat is transported inside the TBsL, while in the top part, a significant part of the heat is transferred from the TBsL toward the center of the channel at a rate $\alpha q_{w}$. Hence, using the conditional means of the maximum temperature location, $\left\langle x_{T} \mid \theta_{w}\right\rangle$, and of the local and 
maximum wall temperatures, $\left\langle\Delta T_{w} \mid \theta_{w}\right\rangle$ and $\left\langle\Delta T_{w, T} \mid \theta_{w}\right\rangle$, one can define dimensionless numbers for each value of $\theta_{w}$. The conditional means of the Nusselt and Rayleigh numbers are plotted in Fig. 8, with $\alpha=1.9$ being chosen to fit the experimental data. The calculation of the error bars is detailed in Appendix A. Below the transition, the Nusselt number is compensated by the following equation, where the exponent differs slightly from that used in Eq. (9a):

$$
N u_{x<x_{T}}^{*}=N u_{T}\left(\frac{R a_{x}}{R a_{T}}\right)^{0.208} \quad\left(x \leq x_{T}\right)
$$

Fig. 8a shows that heat exchange is close to following a one-fifth power law below the transition, which is similar to what was found in [6] for a symmetrically heated channel. Indeed, at the entry of the channel, the flow along the heated plate is not influenced by the presence of the adiabatic plate and behaves like a flow along a single vertical plate. However, in Fig. 8a, the leftmost points highest above the error bar correspond to temperature measurements at the entry $(x / H=0.04)$, where heat losses are greater. Moreover, below the transition, for the lowest values of the average wall temperature $\left(\theta_{w} \lesssim-0.4\right)$, the data depart significantly from Eq. (10) as $\theta_{w}$ decreases (Fig. 8a). More precisely, the data deviate further from Eq. (10) as the maximum wall temperature moves toward the entry, which reduces the length below the transition. As the one-fifth power law is the analytical solution for an infinite vertical plate, it no longer applies when this length becomes too short. Indeed, the deviation is observed for $\theta_{w} \lesssim-0.4$, which corresponds to $\left\langle x_{T} \mid \theta_{w}\right\rangle / H \lesssim 0.5$ (this can be seen on Fig. 6). In the symmetrical case, this deviation was not observed, since the transition was located higher in the channel, at $\left\langle x_{T} \mid \theta_{w}\right\rangle / H=$ 0.7 .

Above the transition, Fig. $8 \mathrm{~b}$ shows that the $N u-R a$ relationship above the transition is modeled by Eq. (9b), which is in line with the results in the symmetrical case. However, a deviation is also observed for the lowest values of the average wall temperature $\theta_{w} \lesssim-0.4$, which show weaker heat transfer than that given by Eq. (9b). To explain this fact, we recall that the heat transfer model (9b), which comes from the symmetrical case, describes the drop in wall temperature observed just above the maximum. In the symmetrical case studied in [6], the channel is too short to allow observation of the expected increase in temperature characterizing a turbulent regime that follows the transition zone of decreasing temperature. Fig. 8b clearly shows that for the lowest values of $\theta_{w}$, the position of the maximum temperature is low enough to reveal the emergence of the turbulent regime characterized by an increase in wall temperature after an initial decrease.

\subsection{Influence of the surroundings}

As already mentioned in Section 3.2, the flow in the cavity probably has an effect on the flow inside the channel. To explore this hypothesis, Fig. 9 shows the conditional means of several quantities as functions of $\theta_{w}$. The lengths of the vertical bars are the corresponding conditional RMs values. Fig. 9a shows the conditional mean of the position of the transition, $\left\langle x_{T} \mid \theta_{w}\right\rangle$, which is the height corresponding to the maximum wall temperature and moves toward the exit as $\theta_{w}$ increases. Fig. $9 \mathrm{~b}$ shows the conditional mean of the wall temperature at the transition point, $\left\langle\Delta T_{w, T} \mid \theta_{w}\right\rangle$, which is defined as the maximum wall temperature. This quantity increases with $\theta_{w}$, which can also be seen in Fig. 6a. The conditional mean of the vertical bulk velocity at $x / H=$ 0.75, $\left\langle\langle U\rangle_{y} \mid \theta_{w}\right\rangle$, shown in Fig. 9c, is a decreasing function of the average wall temperature, with a negative bulk velocity being seen for the highest values of $\theta_{w}$. The velocity is made dimensionless with a typical velocity $U_{I}=(\kappa / H) R a_{H}^{2 / 5}$, which is the order of magnitude of the 


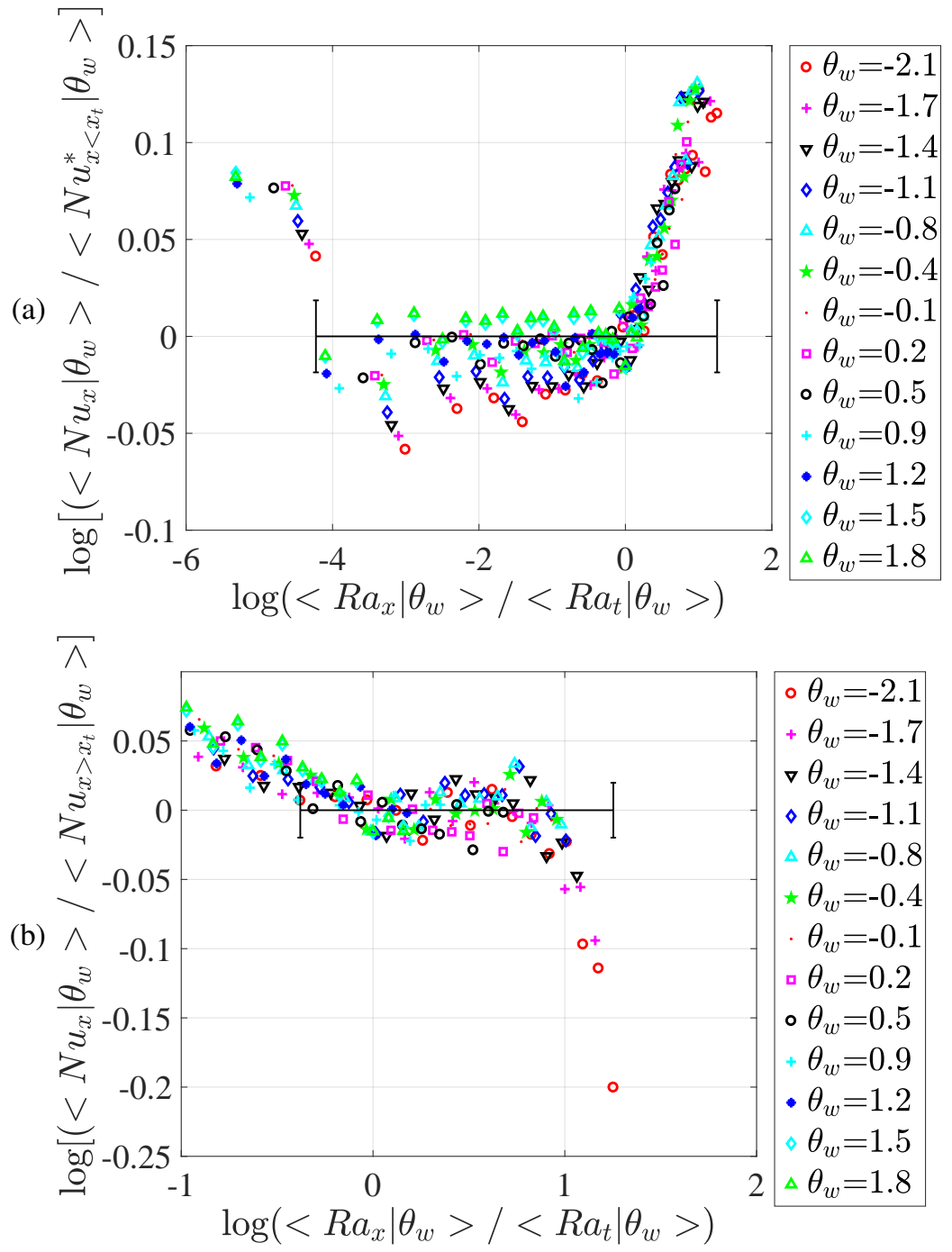

Figure 8: Nusselt-Rayleigh number diagrams conditioned by the value of the average wall temperature. The origin of the horizontal axis corresponds to $x=\left\langle x_{T} \mid \theta_{w}\right\rangle$. The Nusselt number is compensated by the conditional means of (a) $N u_{x<x_{T}}^{*}$ (Eq. (10)) and (b) $N u_{x>x_{T}}$ (Eq. (9b)) 
(a)

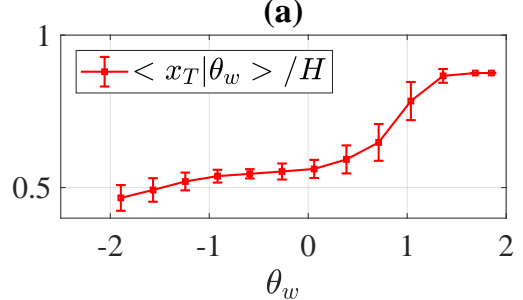

(c)

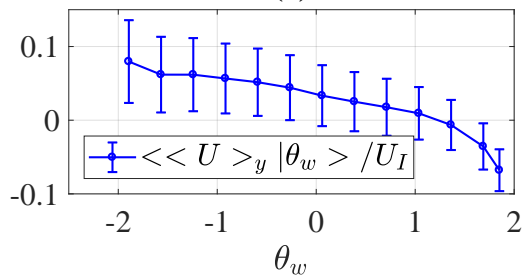

(e)

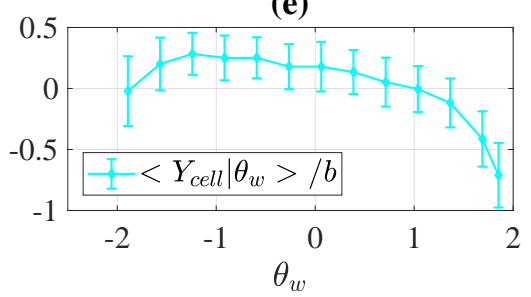

(b)

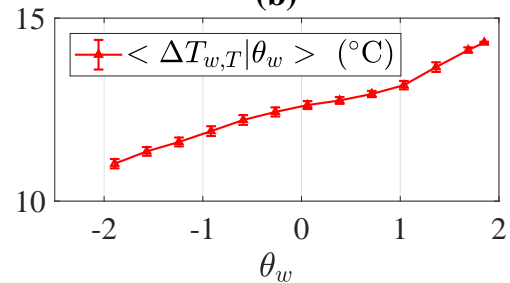

(d)

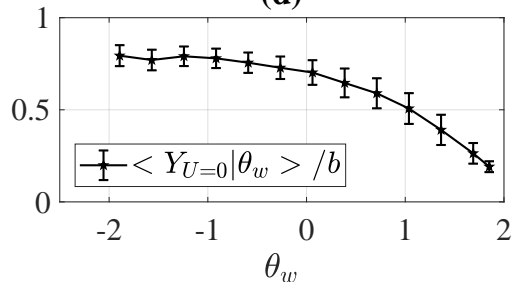

(f)

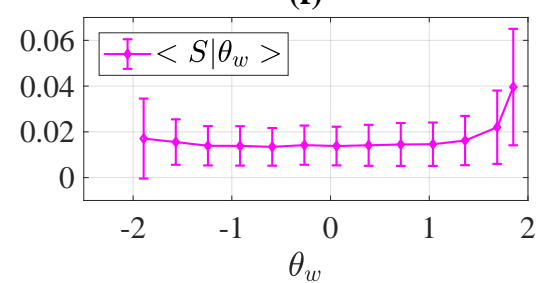

Figure 9: Conditional means of several quantities as functions of the reduced wall temperature $\theta_{w}$. The lengths of the vertical bars are the corresponding conditional Rms values. (a) Position of the maximum wall temperature. (b) Maximum wall temperature. (c) Dimensionless $y$-average vertical velocity at $x / H=0.75$. (d) Spanwise position corresponding to $U=0$. (e) Spanwise position of the plane separating the counter-rotating cells above the channel. (f) External stratification parameter $S$, Eq. (11). See the text for details. 
velocity at the top of a single vertical heated plate obtained from the laminar solution given by Sparrow and Gregg [7] $\left(U_{I}\right.$ is also the velocity scale given in Eq. (4b) in [6]). $\left\langle Y_{U=0} \mid \theta_{w}\right\rangle / b$ (Fig. 9d) is the distance from the heated wall of the zero-vertical-velocity point measured at $x / H=0.75$ divided by the channel width $b$. Thus, $b-\left\langle Y_{U=0} \mid \theta_{w}\right\rangle$ is a typical width of the flow reversal. $\left\langle Y_{\text {cell }} \mid \theta_{w}\right\rangle / b$ (Fig. 9e) is the $y$ position of the plane separating the counter-rotating cells above the outlet of the channel divided by $b$. It is defined as the location of the maximum of the conditional mean of the vertical velocity at $x / H=1.08 .\left\langle S \mid \theta_{w}\right\rangle$ (Fig. 9f) is the conditional mean of an external stratification parameter $S$ representing the pressure variation due to thermal stratification. More precisely, the integral term $\rho g \beta \int_{0}^{H} \Delta T_{e} \mathrm{~d} x$ is the pressure variation over the height of the channel behind the heated plate due to thermal stratification (see Appendix B for details). In order to gauge the magnitude of this term, it is compared with the pressure difference that would result from a linear temperature gradient $\Delta T_{I} / H$, where $\Delta T_{I}=\left(q_{w} H / \lambda\right) R a_{H}^{-1 / 5}$ is the order of magnitude of the temperature at the top of a single vertical heated plate obtained from the laminar solution given by Sparrow and Gregg [7] $\left(\Delta T_{I}\right.$ is also the temperature scale given by Eq. (4c) in [6]). Thus, the stratification parameter is defined as

$$
S=\frac{2}{H \Delta T_{I}} \int_{0}^{H} \Delta T_{e} \mathrm{~d} x
$$

Due to the temperature uncertainty, the values of $S$ in the range $-1.7 \leq \theta_{w} \leq 1.2$ are not significant. Fig. 9f shows that a small increase in the stratification is detected for the highest value of $\theta_{w}$.

The conditional means plotted in Fig. 9 show that for the highest values of $\theta_{w}$, the wall temperature is greatest, with a maximum at the outlet of the channel, the bulk velocity is negative, the flow reversal zone is widest and corresponds to the leftmost position of the counter-rotating cells above the outlet, and the external stratification exhibits a significant increase. It is worth noting that for each value of $\theta_{w}$, the conditional RMs (represented by the length of the vertical bars) is not very large, indicating a relatively small scattering of each conditional distribution. These figures show that the flow experiences multiple states. Most of these states are not observed for long durations, except for the highest value of $\theta_{w}$, which can clearly be seen for $40 \mathrm{~min}$ between $t=222.7 \mathrm{~h}$ and $223.4 \mathrm{~h}$ in Fig. 3. The fact that this state remains observable for a long time can be attributed to external thermal stratification. Indeed, the occurrence of a large flow reversal reduces the flow rate in the channel, favoring the occurrence of thermal stratification outside the channel, which also counteracts the effect of buoyancy. Therefore, the unsteady flow is sometimes locked into the single-plate mode.

With regard to the choice of the average wall temperature as the parameter defining the conditional mean operator, Fig. 9 shows that the conditional means of the maximum wall temperature, the bulk velocity, and the width of the recirculation zone all vary monotonically with $\theta_{w}$. Therefore, any of these quantities could be used instead of $\theta_{w}$. In contrast, if an inappropriate parameter was chosen to define the conditional mean operator, then the resulting global parameters would show no variation with the average wall temperature. Whatever unstable phenomenon is driving the highly unsteady flow is not known, but it is likely that external conditions are somehow responsible. Indeed, one can suppose that the hot fluid at the outlet behaves as a thermal plume in isothermal surroundings. A study by Atmane et al. [19] of natural convection flow around a heated cylinder has shown that interactions between secondary flows and the free surface lead to plume oscillations. Furthermore, a numerical study by Garnier et al. [17] of an asymmetrically heated channel inside a cavity has shown that the direction of the flow at the outlet and the size 


\begin{tabular}{|c|c|c|c|}
\hline Experiment & $b(\mathrm{~mm})$ & $q_{w}\left(\mathrm{~W} \mathrm{~m}^{-2} \mathrm{~K}^{-1}\right)$ & Duration $(\mathrm{h})$ \\
\hline 1 & 30.5 & 3684 & 150 \\
\hline 2 & 40.6 & 3678 & 50 \\
\hline 3 & 49.4 & 3698 & 65 \\
\hline
\end{tabular}

Table 1: Data from experiments with several channel widths $b$.

of the enclosure both have a strong influence on global recirculation in the channel. Therefore, we suggest that the lateral movement of the recirculating cells at the outlet of the channel is responsible for the unsteady behavior observed in this study. Indeed, such movement results in an unsteady pressure profile at the exit of the channel, which will have an effect on global quantities such as the rate and structure of the flow by favoring flow reversal. This is clearly a topic that deserves further investigation.

\subsection{Dependence of heat transfer on channel width}

Experiments (see Table 1) have also been performed with three different channel widths $b$ (Experiment 2 was performed previously).

The correlations given in Eqs. (9) show that the dependence on the channel width lies in one of the parameters $\alpha, R a_{T}$, or $N u_{T}$. Fig. 10 shows the conditional Nusselt number compensated by Eqs. (9) versus the conditional Rayleigh number for the experiments listed in Table 1. For the three aspect ratios, most of the points follow the scaling given by Eqs. (9) with $\alpha=1.9$, with the error bars being the same as those in Fig. 8 (see Appendix A for details). Below the transition (Fig. 10a), the data corresponding to the entry of the channel $x / H=0.04$ (full symbols) are outside the error bars owing to additional heat losses at the entry. In the bottom part of the channel $\left(0.04 H<x \leq\left\langle x_{T} \mid \theta_{w}\right\rangle\right)$, the data that fall outside the error bars correspond to the lowest values of $\theta_{w}$, as shown for $b=40.6 \mathrm{~mm}$ in Fig. 8a. Indeed, in that case below the transition, the transition point is close to the entry $\left(\left\langle x_{T} \mid \theta_{w}\right\rangle \sim 0.5 H\right)$, so the solution for an infinite vertical flat plate, Eq. (9a), does not apply. The same observation can be made with regard to the top part of the channel, $x \geq\left\langle x_{T} \mid \theta_{w}\right\rangle$ (Fig. 10b), where the data corresponding to the lowest values of $\theta_{w}$ depart from Eq. (9b). For these values of $\theta_{w}$, the transition zone described by Eq. (9b)) ends below the outlet, and the flow enters the turbulent regime at the top of the channel.

With regard to the dependence on the aspect ratio, Fig. 10 shows that $\alpha$ is independent of $b$. Owing to the compensation in this figure, the influence of the channel width has to be sought in $\left\langle R a_{T} \mid \theta_{w}\right\rangle$ or $\left\langle N u_{T} \mid \theta_{w}\right\rangle$, that is, in the location of the transition $\left\langle x_{T} \mid \theta_{w}\right\rangle$ or in the wall temperature at the transition point $\left\langle\Delta T_{w, T} \mid \theta_{w}\right\rangle$. The conditional means of the Rayleigh and Nusselt numbers, $\left\langle R a_{T} \mid \theta_{w}\right\rangle$ and $\left\langle N u_{T} \mid \theta_{w}\right\rangle$, at the transition point are plotted in Fig. 11 as functions of the average wall temperature $\left\langle\Delta T_{w}\right\rangle_{x}$ for different widths. The insets show the same quantities as functions of the reduced wall temperature $\theta_{w}$. The error bars are calculated in Appendix A. The Rayleigh number at the transition, $\left\langle R a_{T} \mid \theta_{w}\right\rangle$, increases from $10^{12}$ to $1.5 \times 10^{13}$. For $\left\langle\Delta T_{w}\right\rangle_{x} \geq 12.3^{\circ} \mathrm{C}$, $\left\langle R a_{T} \mid \theta_{w}\right\rangle$ becomes constant because the maximum wall temperature reaches $\left\langle x_{T} \mid \theta_{w}\right\rangle=0.88$, which is the highest attainable since there is a decrease in temperature above this position owing to the proximity of the outlet. In this range, the maximum wall temperature $\left\langle\Delta T_{w, T} \mid \theta_{w}\right\rangle$ increases with $\left\langle\Delta T_{w}\right\rangle_{x}$ (see Fig. 9b for Experiment 2), whereas $\left\langle x_{T} \mid \theta_{w}\right\rangle$ has reached its maximum, and the Nusselt number decreases for $\left\langle\Delta T_{w}\right\rangle_{x} \geq 12.3{ }^{\circ} \mathrm{C}$. Fig. 11a shows that the critical Rayleigh number is strongly dependent on the average wall temperature, which is driven by the flow outside the channel, as shown in Section 3.4. This point is discussed in Section 4. The conditional means 



Figure 10: Nusselt-Rayleigh number diagram conditioned by the value of the average wall temperature for the three experiments listed in Table 1 . For each value of $\theta_{w}$, the Nusselt number is compensated by (a) $N u_{x<x_{T}}^{*}$ (Eq. (10)) and (b) $N u_{x>x_{T}}^{*}$ (Eq. (9b) with $\alpha=1.9$ ). Full symbols correspond to the entry of the channel $x / H=0.04$. Owing to compensation, the origin of the horizontal axis is $x=\left\langle x_{T} \mid \theta_{w}\right\rangle$. 



Figure 11: Conditional means of the Rayleigh number (a) and the Nusselt number (b) at the transition point as functions of the mean wall temperature. The insets show the same quantities as functions of the reduced wall temperature.

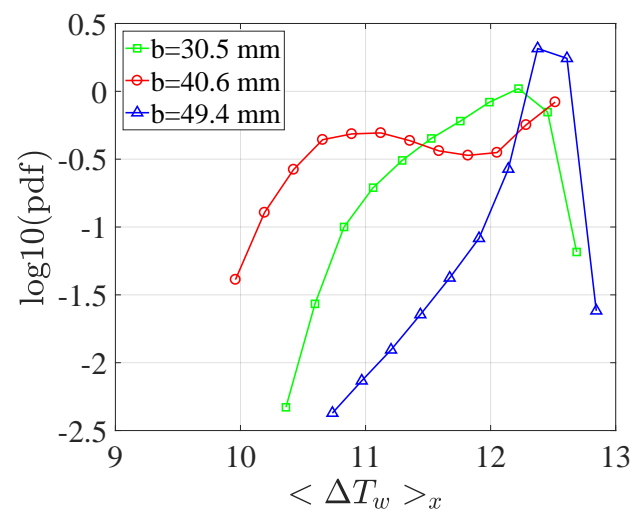

Figure 12: Probability density function (pdf) of the average wall temperature $\left\langle\Delta T_{w}\right\rangle_{x}$ for the three channel widths. Only points that correspond to more than 100 measurements are shown.

of these dimensionless numbers can clearly be seen to be independent of the aspect ratio when they are plotted against $\left\langle\Delta T_{w}\right\rangle_{x}$. As the curves do not merge when they are plotted against $\theta_{w}$, which is constructed from the first and second moments of $\left\langle\Delta T_{w}\right\rangle_{x}(t)$, it can be concluded that the aspect ratio has an effect on the distribution of $\left\langle\Delta T_{w}\right\rangle_{x}(t)$.

Fig. 12 shows the probability density function (pdf) of the average wall temperature for the three experiments listed in Table 1. For large widths, the configuration tends to behave as a single plate, which implies that states with high wall temperature are the most frequent. This trend is also found for the smallest width, which can be explained by the fact that a decrease in width favors laminar flow, so the transition is delayed and the transition point is moved toward the exit. For $b=40.6 \mathrm{~mm}$, the pdf corresponds to a quasi-bimodal distribution, with one mode corresponding to a flow with transition to a turbulent heat flux at mid-height and the other to laminar single-plate flow. These observations show that a transition in an asymmetrically heated channel is observed for all three widths investigated in this study, but its frequency of occurrence 
depends strongly on the aspect ratio $H / b$. The transition is most frequently observed at intermediate aspect ratios. For low aspect ratios (large $b$ ), the configuration moves towards a single heated plate. The fact that the influence of the width can be seen in the distribution of the average wall temperature, which has been shown to be driven by external flows, tends to indicate that the change in the channel width has an impact mainly on the flow outside the channel, as was pointed out in the study by Garnier et al. [17].

\section{Discussion}

This study has shown heat transfer in an asymmetrically heated water channel inside a cavity to be highly unsteady. By using a conditional mean, the average wall temperature is used as a relevant parameter to analyze the flow. This analysis allows a decomposition of the flow into several states that can be analyzed separately. It is important to note that none of these states is sufficiently stable to be observed directly on an experimental bench. Indeed, each average wall temperature value corresponds to a specific wall temperature (Fig. 6a) and a specific vertical velocity profile (Fig. 6b), and most of the quantities shown in Fig. 9 exhibit a monotonic variation with this parameter. Therefore, one may associate a particular flow structure and heat transfer in the channel with a given average wall temperature.

With regard to finding the phenomena that determine a given average wall temperature, the answer is to be sought in the flow outside the channel. In previous work $[4,5,6]$ dealing with a symmetrically heated channel, the flow was found to be statistically steady, with no flow reversal being observed. Therefore, the temperature of the fluid at the inlet was the main parameter characterizing the external flow (providing a sufficiently small thermal stratification in the cavity). With an asymmetrically heated channel, these conclusions do not hold. Considering the glass tank as a thermal system, the thermal boundary conditions that apply to the fluid inside the tank (Fig. 1) are a constant heat flux on the heated plate, constant temperatures along the two diffusers where cold water is discharged, adiabatic conditions on the walls of the glass tank and additional cooling at the free surface that is in contact with ambient air (due to evaporation). Observations show that the temperature of the fluid and the velocity fluctuations at the channel inlet are well controlled, but this is not the case for the flow surrounding the channel. Hence, the motion of the fluid above the channel may favor flow reversals that modify the heat transfer in the channel. As a consequence, the flow in the whole cavity may influence the flow inside the channel, which goes through several states. Thus, the unsteadiness of the flow inside the channel can be attributed to the fluctuations of the flow in the whole cavity, such as the motion of the counter-rotating cell above the channel identified in Section 3.4. Therefore, the average wall temperature can be taken as a parameter that corresponds to a unique flow pattern in the whole tank leading to a unique state of the flow inside the channel.

For each value of the wall temperature, Fig. 11 shows the critical values of the dimensionless numbers corresponding to each state through which the flow passes, the appearances of which are driven by the flow outside the channel. Given that any laboratory experiment on buoyant flow in a vertical channel is inside an enclosure (a cavity or room), it is interesting to note that the threshold defining the transition may be dependent on the surroundings. More generally, the main results depend on the average wall temperature, which becomes a new parameter for studying buoyant flow in vertical channels in unsteady surroundings.

Finally, the results obtained here address the problem of studying the behavior of real systems in uncontrolled surroundings through laboratory experiments. This work has attempted to characterize the buoyant flow inside a vertical channel under various conditions by determining 
the conditional mean of each relevant quantity. If one can obtain the pdf of the average wall temperature that would be obtained under real conditions, then Eq. (6) will give the mean values that would be obtained in real surroundings. Therefore, the next step is to find the distribution or a set of typical distributions of the average wall temperature of a vertical channel in real surroundings.

\section{Conclusions}

This experimental study of natural convection in an asymmetrically heated vertical channel shows that the instantaneous state of the flow depends on the circulations that are observed outside the channel in the entire cavity. However, the conditional mean operator with respect to the average wall temperature allows analysis of each state through which the flow passes. Therefore, the average wall temperature becomes a new parameter for studying buoyant flow in vertical channels in unsteady surroundings. In particular, the distribution of this parameter is key for retrieving meaningful statistical quantities from conditional means. Finally, buoyant flow in a vertical channel in infinite unsteady surroundings may be studied by analyzing a channel in a finite cavity, as long as the external conditions allow a representative range of average wall temperature.

\section{Acknowledgments}

This research was funded by the French National Research Agency in the framework of Project ANR-08-JCJC-0018-01 and the National Agency of Energy Management ADEME-0705C0076. 


\section{Appendix A. Uncertainties calculations}

The aim of this section is to calculate the uncertainties related to the dimensionless numbers which leads to the errorbars plotted on Figs. 8, 10 and 11. Following the method described in Ref. [18], uncertainties of the mean values are first calculated and then the method of propagation of error is used to obtain the uncertainty interval. In this section, uncertainties are calculated for a given parameter $\theta_{w}$ and for clarity the conditional mean operator $\left\langle\cdot \mid \theta_{w}\right\rangle$ is not written.

Let $F$ and $G$ denote the functions used in Figures 8 and 10.

$$
\begin{aligned}
& F=\frac{N u}{N u_{T}}\left(\frac{R a_{T}}{R a_{x}}\right)^{1 / 5} \\
& G=\frac{N u}{N u_{T}}\left(\frac{\alpha+(1-\alpha)\left(\frac{R a_{x}}{R a_{T}}\right)^{1 / 4}}{\left(\frac{R a_{x}}{R a_{T}}\right)^{5 / 4}}\right)^{1 / 5}
\end{aligned}
$$

Using the variables with dimensions, one can write:

$$
\begin{aligned}
& F=\frac{\Delta T_{w, T}}{\Delta T_{w}}\left(\frac{x}{x_{T}}\right)^{1 / 5} \\
& G=\frac{\Delta T_{w, T}}{\Delta T_{w}}\left(\alpha+(1-\alpha) \frac{x}{x_{T}}\right)^{1 / 5}
\end{aligned}
$$

\section{Appendix A.1. Uncertainties in basic variables}

The uncertainty of the mean wall heat flux is described in Section $2: \frac{\mathcal{E}\left(q_{w}\right)}{q_{w}}=1.5 \%$

$x$ is the location of the thermocouple embedded in the heated plate therefore, the uncertainty related to this variable is a fixed error. As the $0.5 \mathrm{~mm}$ thermocouples are glued in a $1 \mathrm{~mm}$ wide groove, the bias limit of $x$ is estimated to $B(x)=0.5 \mathrm{~mm}$ meaning that the real location is within the uncertainty interval $x \pm B(x)$.

The fixed error of $T_{w}$ is attributed to the calibration which leads to the limit bias $B_{1}\left(T_{w}\right)=$ $0.1 \mathrm{~K}$, and to the technical solution used to stuck the sensor on the plate $B_{2}\left(T_{w}\right)$. The latter bias leads to differences in measurements (mainly due to thermal contact between the sensor and the plate) at two different $x . B_{2}\left(T_{w}\right)$ is difficult to measure and in this study it is estimated from the temperature profile along the plate (Fig. 6a) which exhibits non-physical variation between two consecutive thermocouples: $B_{2}\left(T_{w}\right)=0.25 \mathrm{~K}$. Using the root-sum-square (Rss) method, one obtains the overall bias limit:

$$
B\left(T_{w}\right)=\left(B_{1}\left(T_{w}\right)^{2}+B_{2}\left(T_{w}\right)^{2}\right)^{1 / 2}=0.27 \mathrm{~K} .
$$

At the entry, the overall bias is the one due to the calibration: $B\left(T_{\text {inlet }}\right)=0.1 \mathrm{~K}$. Using the propagation of error method (see Eq.16 in Ref. [18]), one can find the bias on $\Delta T_{w}=T_{w}-T_{\text {inlet }}$.

$$
B\left(\Delta T_{w}\right)=\left(B\left(T_{w}\right)^{2}+B\left(T_{\text {inlet }}\right)^{2}\right)^{1 / 2}=0.29 \mathrm{~K} .
$$

Concerning the random error of $\Delta T_{w}$ which is a mean value over the time, it is estimated from the standard deviation $\sigma_{\Delta T_{w}}$. Let $N$ denote the number of values used to estimated $\Delta T_{w}$, the precision index is

$$
S\left(\Delta T_{w}\right)=\frac{\sigma_{\Delta T_{w}}}{\sqrt{N}}
$$


Using the Student's multiplier for $95 \%$ confidence $\eta$, one can calculate the uncertainty on $\Delta T_{w}$ :

$$
\mathcal{E}\left(\Delta T_{w}\right)=\left[B\left(\Delta T_{w}\right)^{2}+\left(\eta S\left(\Delta T_{w}\right)\right)^{2}\right]^{1 / 2} .
$$

For all $\theta_{w}$ and $x$, the random error is found to be negligible with respect to the fixed error.

The uncertainty of the height of the transition $x_{T}$ is $\mathcal{E}\left(x_{T}\right)=12.5 \mathrm{~mm}$ owing to the spatial resolution of the wall-temperature measurements.

Concerning the wall-temperature at the transition, the sources of uncertainties are the same as those of $\Delta T_{w}$ as well as the uncertainty due to the resolution of the wall-temperature measurements. Let $d \Delta T_{w, T}$ and $d x_{T}$ denote variations in $\Delta T_{w, T}$ and $x_{T}$, respectively. One may write:

$$
\begin{aligned}
d \Delta T_{w, T} & =\left.\frac{\partial \Delta T_{w}}{\partial x}\right|_{x=x_{T}} d x_{T} \\
& =\frac{1}{5} \frac{\Delta T_{w, T}}{x_{T}} d x_{T}
\end{aligned}
$$

where the second equation is obtained from the temperature profile in the bottom part (Eq. (9a)). Replacing $d x_{T}$ by $\mathcal{E}\left(x_{T}\right)$ leads to the uncertainty in $\Delta T_{w, T}$ :

$$
\frac{\mathcal{E}\left(\Delta T_{w, T}\right)}{\Delta T_{w, T}}=\left[\left(\frac{B\left(\Delta T_{w, T}\right)}{\Delta T_{w, T}}\right)^{2}+\left(\eta \frac{S\left(\Delta T_{w, T}\right)}{\Delta T_{w, T}}\right)^{2}+\left(\frac{1}{5} \frac{\mathcal{E}\left(x_{T}\right)}{x_{T}}\right)^{2}\right]^{1 / 2} .
$$

$\frac{\mathcal{E}\left(\Delta T_{w, T}\right)}{\Delta T_{w, T}}$ is found to be close to $\frac{\mathcal{E}\left(\Delta T_{w}\right)}{\Delta T_{w}}$.

\section{Appendix A.2. Propagation of error}

Following the propagation of error method given in Ref. [18], the uncertainties on $F$ and $G$ are:

$$
\begin{aligned}
\left(\frac{\mathcal{E}(F)}{F}\right)^{2} & =\left(\frac{\mathcal{E}\left(\Delta T_{w, T}\right)}{\Delta T_{w, T}}\right)^{2}+\left(\frac{\mathcal{E}\left(\Delta T_{w}\right)}{\Delta T_{w}}\right)^{2}+\frac{1}{25}\left[\left(\frac{\mathcal{E}(x)}{x}\right)^{2}+\left(\frac{\mathcal{E}\left(x_{T}\right)}{x_{T}}\right)^{2}\right] \\
\left(\frac{\mathcal{E}(G)}{G}\right)^{2} & =\left(\frac{\mathcal{E}\left(\Delta T_{w, T}\right)}{\Delta T_{w, T}}\right)^{2}+\left(\frac{\mathcal{E}\left(\Delta T_{w}\right)}{\Delta T_{w}}\right)^{2} \\
& +\frac{1}{25\left(1+\frac{\alpha}{1-\alpha} \frac{x_{T}}{x}\right)^{2}}\left[\left(\frac{\mathcal{E}(x)}{x}\right)^{2}+\left(\frac{\mathcal{E}\left(x_{T}\right)}{x_{T}}\right)^{2}\right]
\end{aligned}
$$

In order to simplify the expression of $G$, the factor involving $\alpha$ is taken as a constant: $1+\alpha x_{T} /(1-$ $\alpha) x \simeq-0.5$ which is obtain by considering the value of $\alpha=1.9$ and the mean value $x_{T} / x \simeq 0.7$. The uncertainties $\mathcal{E}(F)$ and $\mathcal{E}(G)$ are calculated for each value of $\left(\theta_{w}, x\right)$. For a given height $x$, the uncertainties are defined as the maximum over $\theta_{w}: \hat{\mathcal{E}}(F) \doteq \max _{\theta_{w}}[\mathcal{E}(F)]$ and $\hat{\mathcal{E}}(G) \doteq \max _{\theta_{w}}[\mathcal{E}(G)]$. They are found to be almost independent on $x$.

$$
\begin{aligned}
& \frac{\hat{\mathcal{E}}(F)}{F}=4.3 \% \\
& \frac{\hat{\mathcal{E}}(G)}{G}=4.6 \%
\end{aligned}
$$


The uncertainty intervals plotted in Figs. $8 \mathrm{a}$ and $10 \mathrm{a}$ are $\pm \frac{\hat{\mathcal{E}}(F)}{F}$ whereas those in Figs. $8 \mathrm{~b}$ and $10 \mathrm{~b}$ are $\pm \frac{\hat{\mathcal{E}}(G)}{G}$.

In the same way, the uncertainty of $R a_{T}$ and $N u_{T}$ are:

$$
\begin{aligned}
& \left(\frac{\mathcal{E}\left(R a_{T}\right)}{R a_{T}}\right)^{2}=\left(\frac{\mathcal{E}\left(\Delta T_{w, T}\right)}{\Delta T_{w, T}}\right)^{2}+\left(4 \frac{\mathcal{E}\left(x_{T}\right)}{x_{T}}\right)^{2} \\
& \left(\frac{\mathcal{E}\left(N u_{T}\right)}{N u_{T}}\right)^{2}=\left(\frac{\mathcal{E}\left(q_{w}\right)}{q_{w}}\right)^{2}+\left(\frac{\mathcal{E}\left(\Delta T_{w, T}\right)}{\Delta T_{w, T}}\right)^{2}+\left(\frac{\mathcal{E}\left(x_{T}\right)}{x_{T}}\right)^{2}
\end{aligned}
$$

These relative uncertainties are found to be almost independent on $b$ and to decrease as $\theta_{w}$ increases. For the lowest values of $\theta_{w}$,

$$
\frac{\mathcal{E}\left(R a_{T}\right)}{R a_{T}} \simeq 16 \% \quad \frac{\mathcal{E}\left(N u_{T}\right)}{N u_{T}} \simeq 5.1 \%,
$$

and for the highest vales of $\theta_{w}$, one obtains:

$$
\frac{\mathcal{E}\left(R a_{T}\right)}{R a_{T}} \simeq 9.5 \% \quad \frac{\mathcal{E}\left(N u_{T}\right)}{N u_{T}} \simeq 3.4 \%
$$

The uncertainty intervals plotted in Figs. 11 are $\pm \frac{\mathcal{E}\left(R a_{T}\right)}{R a_{T}}$ and $\pm \frac{\mathcal{E}\left(N u_{T}\right)}{N u_{T}}$

\section{Appendix B. Pressure variation induced by external thermal stratification}

Thermal stratification of the surroundings has been mentioned as a possible driving parameter for the transition in a symmetrically heated channel (see Section 5 in [6]), and so the conditional means of the temperature profiles measured outside the channel behind the heated plate (the locations of the thermocouples are shown in Fig. 2) are plotted in Fig. B.13. Owing to the uncertainty in temperature measurement, the profiles corresponding to $-1.7 \leq \theta_{w} \leq 1.2$ are within the error bar, whereas the difference in temperature measured at the exit and entry heights for the greatest values of $\left|\theta_{w}\right|$ are significant. These temperature differences are significant despite their small values, since their impact on the pressure difference cannot be ignored. Indeed, for the symmetrical configuration studied with the same apparatus in [6], just a few tenths of a degree of external thermal stratification leads to an external pressure difference that is equivalent to that estimated inside the channel. To quantify this effect, it is useful to characterize the thermal stratification in terms of pressure difference instead of temperature difference. Let $\overrightarrow{U_{e}}, \overrightarrow{u_{e}^{\prime}}, T_{e}$, and $P_{e}$ denote the mean velocity vector, the velocity fluctuation, the mean temperature, and the mean pressure outside the channel. Using the Oberbeck-Boussinesq approximation, the density of the fluid at a temperature $T$ is written as $\rho\left[1-\beta\left(T-T_{\text {ref }}\right)\right]$, where $\rho$ is the density at the reference temperature $T_{\text {ref }}$. We define also $\hat{P}_{e}=P_{e}+\rho g \beta\left(T_{\text {inlet }}-T_{\text {ref }}\right)$. Assuming statistically stationary flow, the scalar product of $\vec{U}_{e}$ with the momentum equation of the mean flow leads to

$$
\overrightarrow{U_{e}} \cdot \vec{\nabla}\left[\frac{1}{2}{\overrightarrow{U_{e}}}^{2}+\frac{\hat{P}_{e}}{\rho}+g x\right]=v \overrightarrow{U_{e}} \cdot \Delta \overrightarrow{U_{e}}-\overrightarrow{U_{e}} \cdot\left(\nabla\left\langle\overrightarrow{u_{e}^{\prime}} \otimes \overrightarrow{u_{e}^{\prime}}\right\rangle_{t}\right)+g \beta \Delta T_{e} \overrightarrow{U_{e}} \cdot \vec{x}
$$

where $\nabla=(\partial / \partial x, \partial / \partial y, \partial / \partial z)$ is the gradient operator, $\Delta T_{e}=T_{e}-T_{\text {inlet }}$ is the temperature difference with respect to the inlet temperature, and $\cdot$ and $\otimes$ are the dot and tensor products. The 


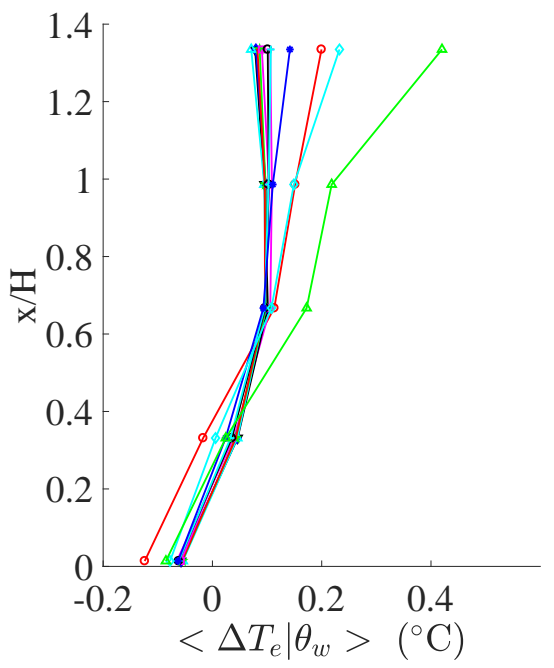

$$
\begin{aligned}
& -\theta_{w}=-2.1 \\
& -\theta_{w}=-1.7 \\
& \rightarrow \theta_{w}=-1.4 \\
& -\theta_{w}=-1.1 \\
& -\theta_{w}=-0.8 \\
& -\theta_{w}=-0.4 \\
& -\theta_{w}=-0.1 \\
& -\theta_{w}=0.2 \\
& -\theta_{w}=0.5 \\
& -\theta_{w}=0.9 \\
& \rightarrow \theta_{w}=1.2 \\
& -\theta_{w}=1.5 \\
& -\theta_{w}=1.8
\end{aligned}
$$

Figure B.13: Conditional mean of the temperature profile outside the channel behind the heated plate. $x=0$ is the heigh of the entry. The locations of the sensors are shown in Fig. 2.

left-hand side is the directional derivative of the term inside the square brackets. The terms of the right-hand side represent the dissipation of momentum by molecular viscosity, the interaction between the mean flow and the fluctuations, and the power of the buoyancy force. For strictly steady and isothermal flow of an inviscid fluid, the right-hand side is equal to zero and one finds the classical form of Bernoulli's equation along a streamline. To go further, let us suppose that the external temperature depends only on the height: $\Delta T_{e}=f(x)$. Then Eq. (B.1) can be written as

$$
\overrightarrow{U_{e}} \cdot \vec{\nabla}\left[\frac{1}{2}{\overrightarrow{U_{e}}}^{2}+\frac{\hat{P}_{e}}{\rho}+g x-g \beta \int_{0}^{x} \Delta T_{e} \mathrm{~d} x\right]=v \overrightarrow{U_{e}} \cdot \Delta \overrightarrow{U_{e}}-\overrightarrow{U_{e}} \cdot\left(\nabla\left\langle\overrightarrow{u_{e}^{\prime}} \otimes \overrightarrow{u_{e}^{\prime}}\right\rangle_{t}\right)
$$

Therefore, the integral term $\rho g \beta \int_{0}^{H} \Delta T_{e} \mathrm{~d} x$ is the pressure variation over the height of the channel behind the heated plate due to thermal stratification.

\section{References}

[1] A. Bar-Cohen, W. M. Rohsenow, Thermally optimum spacing of vertical, natural convection cooled, parallel plates, Journal of Heat Transfer 106 (1984) 116-123.

[2] M. Miyamoto, Y. Katoh, J. Kurima, H. Sasaki, Turbulent free convection heat transfer from vertical parallel plates, in: Hemisphere (Ed.), International Heat Transfer Conference, Vol. 4, 1986, pp. 1593-1598.

[3] B. W. Webb, D. P. Hill, High Rayleigh number laminar natural convection in an asymmetrically heated vertical channel, Journal of Heat Transfer 111 (1989) 649-656.

[4] C. Daverat, H. Pabiou, C. Ménézo, H. Bouia, S. Xin, Experimental investigation of turbulent natural convection in a vertical water channel with symmetric heating: Flow and heat transfer, Experimental Thermal and Fluid Science 44 (2013) 182-193. doi:10.1016/j.expthermflusci.2012.05.018.

[5] C. Daverat, Y. Li, H. Pabiou, C. Ménézo, S. Xin, Transition to turbulent heat transfer in heated vertical channelExperimental analysis, International Journal of Thermal Sciences 111 (2017) 321-329.

[6] Y. Li, C. Daverat, H. Pabiou, C. Ménézo, S. Xin, Transition to turbulent heat transfer in heated vertical channelScaling analysis, International Journal of Thermal Sciences 112 (2017) 199-210. 
[7] E. M. Sparrow, J. L. Gregg, Laminar free convection from a vertical plate with uniform surface heat flux, Transaction of the ASME 78 (1956) 435-440.

[8] C. K. Liu, G. C. Vliet, An experimental study of turbulent natural convection boundary layers, Journal of Heat Transfer 91 (1969) 517-531.

[9] E. M. Sparrow, G. M. Chrysler, L. F. Azevedo, Observed flow reversals and measured-predicted nusselt numbers for natural convection in a one-sided heated vertical channel, Journal of Heat Transfer 106 (1984) 325-332.

[10] S. Ostrach, An analysis of laminar free-convection flow and heat transfer about a flat plate parallel to the direction of the generating body force, Tech. Rep. 2635, NACA (1952).

[11] E. Sanvicente, S. Giroux, C. Ménézo, H. Bouia, Transitional natural convection flow and heat transfer in an open channel, International Journal of Thermal Sciences 63 (2013) 87-104.

[12] F. Dupont, F. Ternat, S. Samot, R. Blonbou, Two-dimension experimental study of the reverse flow in a free convection channel with active walls differentially heated, Experimental Thermal and Fluid Science 47 (2013) $150-157$.

[13] W.-S. Fu, S.-H. Huang, C.-G. Li, An investigation of unsteady flow reversal of natural convection in vertical parallel plates by the modified local one-dimensional inviscid relations method, International Journal of Heat and Mass Transfer 86 (2015) 124-138.

[14] W.-S. Fu, W.-S. Chao, T.-E. Peng, C.-G. Li, Flow downward penetration of vertical parallel plates natural convection with an asymmetrically heated wall, International Communications in Heat and Mass Transfer 74 (2016) 55-62. doi:10.1016/j.icheatmasstransfer.2016.03.006.

[15] G. Polidori, S. Fatnassi, R. B. Maad, S. Fohanno, F. Beaumont, Early-stage dynamics in the onset of free-convective reversal flow in an open-ended channel asymmetrically heated, International Journal of Thermal Sciences 88 (2015) 40-46.

[16] D. Ospir, C. Popa, C. Chereches, G. Polidori, S. Fohanno, Flow visualization of natural convection in a vertical channel with asymmetric heating, International Communications in Heat and Mass Transfer 39 (2012) 486-493.

[17] C. Garnier, A. Sergent, Y. Fraigneau, P. Le Quéré, Comparative study of numerical simulations of a 2D buoyancydriven flow in a vertical channel asymmetrically heated with or without external domain, in: Proceedings of the 15th international heat transfer conference. Kyoto, Japan IHTC15-9291, 2014.

[18] R. J. Moffat, Describing the Uncertainties in Experimental Results, Experimental Thermal and Fluid Science, 1 (1988) 3-17.

[19] M. A. Atmane, V. S. Chan, D. B. Murray, Natural convection around a horizontal heated cylinder: the effects of vertical confinement, International Journal of Heat and Mass Transfer 46 (19) (2003) 3661-3672.

[20] S. Pope, Turbulent flows, Cambridge University Press, Cambridge, 2000 